(ages 1-5 years: adjusted hazard ratio [AHR] for each additional week of gestation, 0.86 [95\% CI, 0.80-0.94], $P<.001$, based on 120 deaths), which weakened and was nonsignificant in later childhood (ages 6-12 years: AHR, 0.90 [95\% CI, 0.73-1.10], $P=.31$, based on 21 deaths), adolescence (ages 13-17 years: AHR, 0.92 [95\% CI, 0.75-1.11], $P=.38$, based on 25 deaths), and young adulthood (ages 18-36 years: AHR, 0.90 [95\% CI, 0.81-1.01], $P=.07$, based on 77 deaths). When the latter 3 age ranges were combined to improve statistical power, there was a significant inverse association between gestational age at birth and infection-related mortality in this broader age range (ages 6-36 years: AHR, 0.91 [95\% CI, 0.83-0.99], $P=.02$ ).

We also reexamined the association between gestational age at birth and respiratory mortality after excluding infection-related deaths (ie, censoring these individuals at the time of death). In early childhood, low gestational age at birth was no longer associated with respiratory mortality (ages 1-5 years: AHR for each additional week of gestation, 1.06 [95\% CI, 0.82-1.36], $P=.65)$. However, in young adulthood, gestational age at birth remained inversely associated with respiratory mortality (ages 18-36 years: AHR, 0.82 [95\% CI, 0.71-0.95], $P=.009$ ). This association in young adulthood was due to various diagnoses, of which the most common were asthma, ${ }^{1}$ pneumonitis, other interstitial pulmonary diseases, and pulmonary edema.

We also assessed the sensitivity of our all-cause mortality results to infection-related deaths by repeating our main analyses after censoring all 243 of these deaths. The risk estimates in all age ranges (as reported in Table 2 of our article) remained virtually identical.

These supplemental findings suggest that gestational age at birth has a strong inverse association with infection-related mortality in early childhood, which weakens but may not completely resolve in later childhood through young adulthood. In young adulthood, low gestational age at birth was associated with increased respiratory mortality even after excluding infectionrelated deaths. Additional epidemiological and mechanistic studies of the effect of preterm birth on immune function and infection risk throughout the life course are warranted.

Casey Crump, MD, PhD

Kristina Sundquist, MD, PhD

Jan Sundquist, MD, PhD

Author Affiliations: Department of Medicine, Stanford University, Stanford California (Dr Crump) (kccrump@stanford.edu); and Center for Primary Health Care Research, Lund University, Malmö, Sweden (Drs K. Sundquist and J. Sundquist)

Conflict of Interest Disclosures: All authors have completed and submitted the ICMJE Form for Disclosure of Potential Conflicts of Interest and reported grant support from the National Institute of Child Health and Human Development, the Swedish Research Council, the Swedish Council for Working Life and Social Research, and ALF project grant, Lund, Sweden.

1. Crump C, Winkleby MA, Sundquist J, Sundquist K. Risk of asthma in young adults who were born preterm: a Swedish national cohort study. Pediatrics. 2011; 127(4):e913-e920.

\section{Time-Limited Trials}

To the Editor: The Commentary on time-limited trials (TLTs) of treatment by Drs Quill and Holloway underscores the need for an explicit process to better manage "major interventions in circumstances in which the outcome is uncertain." "The basic tenets of the authors' proposal included measurement of end points; agreement on a time point for reevaluation of an individual's therapeutic response; and reconsideration of the intervention, with possible continuation, dose reduction, or cessation based on response.

This process outlined for TLTs should pertain to all clinical interventions, not simply to "major interventions" or to those provided to people near the end of life. Having a process in place to ensure net clinical benefit (ie, a balance between clinical response and toxicity) is important if iatrogenic harm is to be minimized, patient burden reduced, and health care costs contained. Clinicians must consistently ask "Should this intervention be continued?" This evaluative process will require an explicit set of measures matched to defined outcomes. Clinicians should define and discuss the therapeutic goals of a new intervention before reaching for the prescription pad.

There is a research agenda corollary to this clinical motivation. A clear understanding of therapeutic intent requires knowledge of the targeted level of prevention and the time frame within which an individual's benefit should accrue. ${ }^{2}$ Consider statins for secondary prevention of cardiovascular disease. Should a person with metastatic lung cancer continue taking statins? Using principles of comparative effectiveness research, carefully designed TLTs could generate evidence to inform such decisions; these trials' results could apply to both population and patient levels, informing both when to initiate therapies and when to stop them. These studies are in their infancy. ${ }^{3,4}$

Physicians and patients require practical information to guide decision making. Time-limited trials mirror more formal n-of- 1 trials that evaluate therapies objectively but are often clinically infeasible. The ultimate in personalized medicine, n-of-1 studies entail blinded evaluation of repeated cycles alternating between therapies. Certain therapies are ill-suited to this evaluation, particularly those with long delays in onset of action or very long half-lives; n-of-1 trials are inappropriate for patients with unstable disease. ${ }^{5}$ Lacking the logistical challenges of n-of- 1 studies, TLTs offer an explicit process more suitable for guiding routine practice.

Amy P. Abernethy, MD

David C. Currow, BMed, MPH, FRACP

Author Affiliations: Duke Cancer Institute, Duke University Medical Center, Durham, North Carolina (Dr Abernethy) (amy.abernethy@duke.edu); and Department of Palliative and Supportive Services, Flinders University, Adelaide, Australia (Dr Currow).

Conflict of Interest Disclosures: Both authors have completed and submitted the ICMJE Form for Disclosure of Potential Conflicts of Interest. Dr Abernethy reported that she receives research funding from the US National Institutes of Health, US Agency for Healthcare Research and Quality, Robert Wood Johnson Foundation, Pfizer, Eli Lilly, Bristol-Meyers Squibb, Helsinn Therapeutics, Amgen, Kanglaite, Alexion, Biovex, DARA Therapeutics, Novartis, and Mi-Co; 
these funds are all distributed to Duke University Medical Center to support research. In the last 2 years she has had consulting agreements $(<\$ 10,000)$ with Helsinn Therapeutics, Amgen, Novartis, and Bristol Meyers Squibb. Dr Currow reported no disclosures.

1. Quill TE, Holloway R. Time-limited trials near the end of life. JAMA. 2011; 306(13):1483-1484

2. Stevenson J, Abernethy AP, Miller C, Currow DC. Managing comorbidities in patients at the end of life. BMJ. 2004;329(7471):909-912.

3. Garfinkel D, Mangin D. Feasibility study of a systematic approach for discontinuation of multiple medications in older adults: addressing polypharmacy. Arch Intern Med. 2010;170(18):1648-1654.

4. Abernethy AP, Aziz NM, Basch E, et al. A strategy to advance the evidence base in palliative medicine: formation of a palliative care research cooperative group. J Palliat Med. 2010;13(12):1407-1413.

5. Nikles J, Mitchell G, Walters J, et al. Prioritising drugs for single patient (nof-1) trials in palliative care. Palliat Med. 2009;23(7):623-634.

In Reply: Although our Commentary focused exclusively on TLTs toward the very end of life, we agree with Drs Abernethy and Currow that the concept has much broader applicability. In fact, virtually all interventions with uncertain clinical effects on outcomes could be framed in these terms, in which the probability of success, markers of response, and associated time frames would be presented and agreed upon upfront (eg, nearly all palliative chemotherapy regimens, biological treatments for autoimmune disorders, and all major invasive interventions such as mechanical ventilation, renal dialysis, or feeding tubes).

In addition, as suggested by Abernethy and Currow, treatments with effects that are realized only if one is likely to live a long time (tight control of blood glucose, blood pressure, or cholesterol) have much less relevance (and may even be harmful) as prognosis worsens. In that sense, all medical treatments deserve reevaluation at regular time intervals depending on changes in the patient's condition and prognosis.

Time-limited trials may help us be more realistic, systematic, and regular in reassessments about what medicine can and cannot do, thereby contributing simultaneously to quality improvement and cost control.

Timothy E. Quill, MD

Robert Holloway, MD, MPH

Author Affiliations: Center for Ethics, Humanities, and Palliative Care, University of Rochester Medical Center, Rochester, New York (timothy_quill@urmc.rochester edu).

Conflict of Interest Disclosures: Both authors have completed and submitted the ICMJE Form for Disclosure of Potential Conflicts of Interest. Dr Quill reported receiving payment for lectures on aspects of palliative care and receiving book roy alties. Dr Holloway reported receiving grants from the National Institutes of Health; serving as associate editor of Neurology Today; and serving as a reviewer of neurology guidelines for Milliman Guidelines Inc.

\section{RESEARCH LETTER}

\section{POLST Registry Do-Not-Resuscitate Orders and Other Patient Treatment Preferences}

To the Editor: The Physician Orders for Life Sustaining Treatment (POLST) form augments traditional methods for advance care planning by translating treatment preferences into medical orders, including for cardiopulmonary resuscitation (CPR), scope of treatment, artificial nutrition by tube, and in some states, antibiotic use. Health professionals complete forms based on conversations with willing patients, primarily with advanced illness or frailty, or surrogates. These orders then guide treatment in any setting. In 3 states, POLST forms were effective in influencing the care that patients received. ${ }^{1}$ POLST programs currently exist or are in development in 34 states. $^{2}$ We compared the preferences for other treatments among persons with do-not-resuscitate (DNR) orders and those with attempt CPR orders, using the first year of Oregon POLST Registry data (December 3, 2009, to December 2, 2010).

Methods. POLST includes 2 possible orders for resuscitation: do not attempt CPR (DNR) or attempt CPR. Scopeof-treatment orders consist of limited additional interventions and full treatment, which include hospitalization, and comfort measures only. POLST forms identify patients using name, birth date, sex, and address.

In Oregon, POLST forms are entered into a registry, allowing emergency personnel and hospitals 24-hour access to POLST information when the physical form cannot be located during an emergency. Clinicians in Oregon are required by law to submit forms to the registry unless the patient opts out.

Data were analyzed using IBM SPSS Statistics version 19.0.0 and an online $95 \%$ confidence interval calculator. ${ }^{3}$ The institutional review boards of the Oregon Health \& Science University and Oregon State Public Health approved this study and the waiver of informed consent.

Results. At the end of the first year, 25142 people had active POLST forms in the registry: 85.9\% (21 599/25 142) were aged 65 years or older, $61.0 \%$ ( $14255 / 23376)$ were female, and 40.4\% (8014/19 859) resided in a rural area (denominators $<25142$ reflect missing data). There were $27.9 \%$ (7026/25 142) with an order to attempt CPR and $72.1 \%$ (18 116/25 142) with a DNR order. For patients with an attempt CPR order, $75.7 \%$ (5218/6895) had orders for full treatment, 21.6\% (1492/6895) limited additional interventions, and $2.7 \%$ (185/6895) comfort measures only (TABLE). Of the 18002 patients with a DNR order, 8929 (49.6\%) had orders for comfort measures only and 9073 (50.4\%) had orders for either full treatment or limited additional interventions. Thus, half of patients with DNR orders would want to be transported to the hospital if indicated, and half would not unless comfort needs could not be met in their current location.

Among those with POLST DNR orders, the probabilities for having orders for other life-sustaining treatment ranged from 0.101 to 0.557 except for full treatment and longterm tube feeding (Table). Among those with POLST orders to attempt CPR, the probabilities for having orders for other life-sustaining treatments were higher for 6 of the 9 other orders, ranging from 0.021 to 0.739 .

Comment. The Oregon POLST Registry allows examination of patient preferences beyond resuscitation status. The number of registry submissions in its first year is note- 